\title{
Improving Food safety in Supply Chain based on Big Data
}

\author{
Gang Liu ${ }^{1}$,Guang $\mathrm{Li}^{1, \mathrm{a}}$, Rui Yang ${ }^{1}$, Li Guo ${ }^{1}$ \\ ${ }^{1}$ School of Economy and Management, Tianjin Agricultural University, jinjing road 22 in Xiqing district, Tianjin 300384, china
}

\begin{abstract}
With the rapid development of big data collection and analysis, these tools are increasingly applied to food safety and quality. Big data can play an important role in improving food safety management. This paper will deeply analyze the food safety risk warning system based on big data management. The research results show that the food safety management system based on big data includes data source, data collection and storage, data analysis and application of analysis results.
\end{abstract}

\section{Introduction}

The data and information of food safety are scattered in the whole supply chain, including agricultural sector, processing sector and health sector. As the government and society pay more and more attention to food safety, both information from regulators and enterprises, as well as food safety information from the external environment, are on an explosive growth trend. If the ability to calculate powers is impeding the analysis of food safety monitoring data and vast amounts of external information, then big data technology can make a valid analysis and warning of food safety risk possible. As a new technology leading the future prosperity, big data technology is being applied in the fields of enterprise competition, government management and public service, which have produced great commercial and social value. The food safety early warning system based on big data management is conducive to changing traditional law enforcement and post-reaction of food safety supervision mode and overcoming the disadvantages of the traditional early warning system.

\section{Literature review}

Gartner (2012) as having three-dimensional characteristics, Big Data is high volume, high velocity, and high variety information assets that require new forms of processing to enable enhanced decision making, insight discovery and process optimization. The European Commission (EC) has issued a similar definition (EC, 2014), referencing the three Vs of Volume, Velocity and Variety. Mary Clare Ahearn considers two challenges in the supply chain's response to consumer demand: the use of sustainability metrics and the possibility of improving food safety. Through careful evaluation of the two examples, it is concluded that big data technology is applied to agricultural production. Finally, some comments are made on the potential problems and implications of the application of big data technology in the future of agricultural production. Jing Wang puts forward a food safety early warning system that uses association rule mining and Internet of Things technology. This system can monitor all the testing data of the whole supply chain in time and can automatically warn, which can help food manufacturers to identify food safety risks in advance and provide decision support information. From the perspective of big data, according to the principle of scientific, systematic, operability and analytic hierarchy process Wu Xiangxiang design meat food security early warning index system, which can effectively prevent and control the safety risk of meat food. From the perspective of big data, ZZ zhang is based on food safety forefront of the development of supply chain management, she study the symbiotic mechanism, mode of operation of its business and other aspects of the evolution of trends under the influence of big data, which can improve the theoretical system of food safety supply chain management, give effective guidance to practice, improve the ability of commercial enterprises to use big data and improve the ability of avoid potential risks and adverse effects.

\section{Food safety risk}

Risk generally refers to the possibility of loss, injury or disadvantage. In modern society, risks are increasingly characterized by high uncertainty, unpredictability, and time lag, etc. With the continuous progress of society, the public is more and more sensitive to public safety risks, such as the resistance to large chemical projects near their residential areas and the concern and attention to environmental pollution, etc. Compared with other public safety risks, food safety risks have the following characteristics:

Firstly, food safety risks are more complex. From the perspective of supply chain, food supply chain is composed of raw material supply of agricultural products, farming and breeding of agricultural products,

*Corresponding author: ${ }^{\text {a }}$ 466618280@qq.com 
processing of agricultural products, and the production, distribution, retail and catering of food. Its chain is very long, and any safety problem in any link will bring the food safety hazard of the whole supply chain. Any safety problem in any link will bring the food safety hazard to the whole supply chain. In the production source of agricultural products, the decentralized operation of a large number of farmers has brought great difficulty to the trace ability and quality supervision of agricultural products. At the same time, the quality control model based on sampling inspection and inspection delivery is difficult to restrain the farmers' production behavior, which increases quality risks of agricultural production source represented by pesticide, fertilizer and other excessive residues. The same problem exists in the process flow, the decentralized operation of a large number of small and medium-sized operators has increased the difficulty of supervision, and there is a possibility of food safety risk in processing, logistics, retail and catering.

Secondly, there are many factors influencing food safety risk. Compared with other types of public safety risks, food safety risks are affected by more factors. In the source of agricultural products, residues of agricultural and veterinary drugs, harmful metals, environmental pollution and biological pollution will all bring food safety hazards; In the process of processing, food additives, hazards caused by hot processing, safety problems of new technologies and new resources and the processing environment will all affect food safety; In the process of logistics, food safety will be caused by the health problems of packaging materials, the health problems caused by improper temperature and the pollution of hazardous substances in transportation and storage. In addition, Artificial fake and inferior food is also an important factor of food safety risk.

Thirdly, range of influence of food safety risks is wide. Many public security risks have relatively limited scope and they are generally limited to a particular area, but food safety risk has a relatively large influence scope. With the gradual separation of food production and consumption, as well as the continuous integration of the food industry, a large number of food products have presented a nationwide distribution pattern. At the same time, food safety risks will be continuously transmitted downstream along the food supply chain. In the melamine event, raw milk contained melamine enters the production process of enterprises along the supply chain, then "Problem" milk goes into circulation. With the enterprise's sales network "Problem" milk enter into a number of national markets, which ended up with hurting a lot of consumers.

\section{Food Safety Risk Early Warning Based on Big Data.}

Big data refers to large amounts of different types of data produced with high velocity from a high number of various types of sources. Handling highly variable and real-time data sets requires new tools and methods, such as powerful processors, software and algorithms. Big data can play an important role in food safety early warning. The origins of early warning theory can be traced back to the late 19th century, initially appeared in the field of economics, and then gradually applied to many fields, especially in the field of public safety. Early warning is one of the important ways to avoid the deterioration of public safety incidents. It is a high level warning of the further development of the incident. Risk warning is an important part of risk analysis. The Codex Alimentarius Commission (CAC) considers the risk analysis framework to be the best way to deal with any potential or actual food safety issues, including risk assessment, risk management and risk communication. The basic contents of risk assessment include hazard identification, hazard description, exposure assessment, risk profile. Risk management refers to a series decision making in order to reduce the adverse effects. Risk communication is a shared mechanism with all risk stakeholders. Early warning of food safety refers to the early warning and active prevention of the spread of toxic and harmful substances in food. Food security early warning system can reduce the risk of food safety incidents through the monitoring of food safety problems, tracking, and quantitative analysis, information about forecast. Food safety early warning system has the functions of information delivery, risk communication, forecasting, control and handling etc. The system generally includes information acquisition system, early warning evaluation index system, early warning analysis and decision system, alarm system and warning system, etc. Based on the general process of big data processing, this research builds the framework of early warning of food safety risk based on big data, shown in Figure 1. 


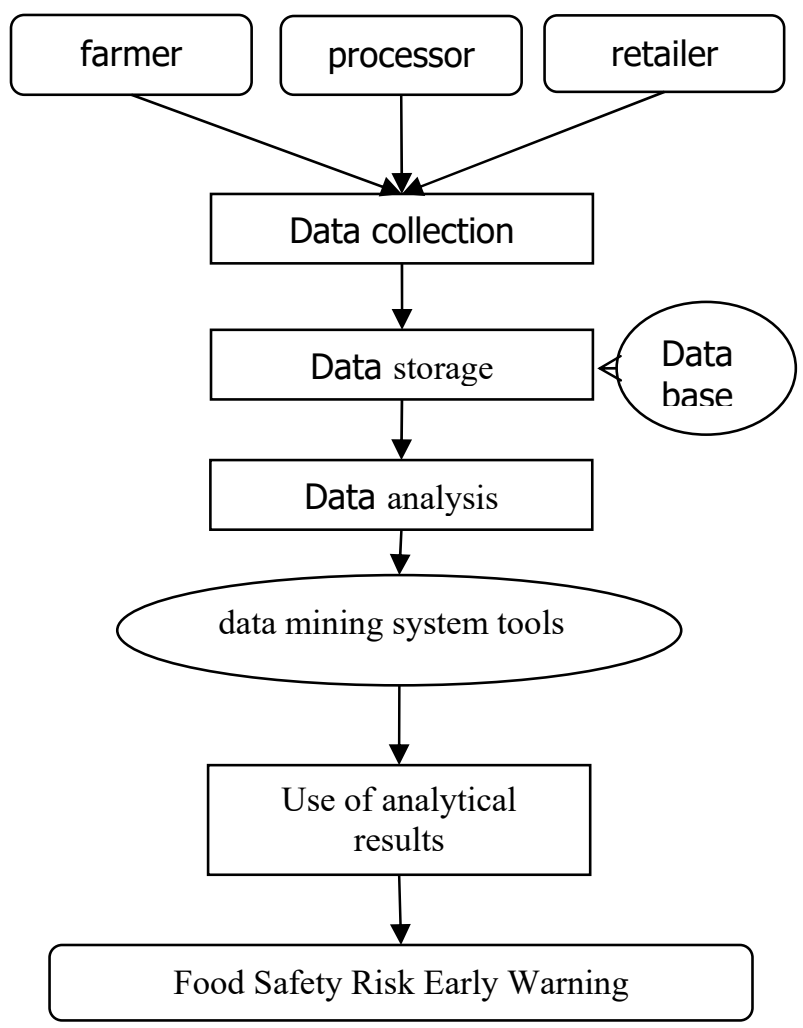

Figure 1. The framework of early warning of food safety risk based on big data

Big data technology can help to extract valuable information from massive food safety information, thus realizing the effective warning of food security risks. Food safety risk information includes regulatory information and intelligence information. Among them, regulatory information includes regulatory standard information, administrative license information, administrative law enforcement information, inspection and detection information, complaint reporting and enterprise self-reported information, etc. Intelligence information is collected in the media, domestic and foreign organizations and other channels, such as the World Health Organization released the news, domestic and foreign news, and internet public opinion and so on.

(1)Data collection

Food safety data has the origin of diversity, frequent updates, data size, data structure and other characteristics. In addition to the structured database of the regulatory system, there are a number of useful information hidden in a large amount of unstructured data. During the data acquisition phase, the data should include food safety trace ability systems and data from the mass media in addition to the regulatory and production sectors.

(2)Data collection, correlation, storage

These include the selection of the key points of food safety regulation, the discovery of the hot spots of public opinion, the sharing of big data resources, the interconnection of information isolated islands, the processing and analysis of structured, semi-structured, unstructured data and streaming media data, and the correlation of big data storage.

(3)Data Analysis

In the data analysis stage, it is necessary to introduce a big data management system and technical process, select appropriate data mining system tools, set up a data warehouse, clean the basic data and establish the data analysis model by using the algorithms and tools such as association and clustering. This paper analyzes the risk level evaluation and early warning mechanism of the risk rating and puts forward some Suggestions on the risk management of food safety.

(4)Use of analytical results

The results of data analysis include public, government and enterprises. Early warning of food safety risks based on big data plays an important role for the government to correctly handle food safety risks, and for the public to properly understand and respond to food safety risks and to promote self-regulation of enterprises and industries.

\section{Conclusions}

From the perspective of frequent food safety incidents, China's existing monitoring network and risk early warning system of food safety have not played an effective role in food safety risk early warning. The first reason is that food safety risks involve a wide range of risks and have many influencing factors. Due to the scarcity of public law enforcement resources, information collection mostly adopts sampling method, which is easy to lead to the omission of risk information. The second is "monitoring network of food safety promotes fast" but "monitoring data processing of food safety is slow, and the phenomenon of data idle and Data Island is serious. In addition, a lot of food safety data lack analysis, which make food safety data lost its warning value; third, the insufficient use of external data, such as internet data, has not made online public opinion and social forces play an important role in food safety risk warning. Managing food safety data can fully excavate the value of mass data, and realize accurate and timely warning of risk of food security. At the same time, managing food safety data can also establish a supervision mode of truth actuation and intelligent control, and improve food safety supervision performance.

\section{Acknowlege}

This work was partially supported by 2018 key entrusted issues from Rural Revitalization Research Institute of Tianjin Agricultural University; also supported by the innovation team training project of colleges and universities in Tianjin in the period of Thirteenth five year plan (TD13-5093); the innovation and entrepreneurship training program for college students in Tianjin (Project No: 201510061029). 


\section{Reference}

1. Marvin HJ, et-al. "Big data in food safety; an overview." C R C Critical Reviews in Food Technology 57.11(2016):2286-2295.

2. Ahearn, Mary Clare, W. Armbruster, and R. Young. "Big Data's Potential to Improve Food Supply Chain Environmental Sustainability and Food Safety." International Food \& Agribusiness Management Review (2016).

3. Wang, Jing, and H. Yue. "Food safety pre-warning system based on data mining for a sustainable food supply chain." Food Control 73(2017):223-229.

4. Cao Chunli, and Business School. "Research on Meat Food Security Early Warning Index System Based on Supply Chain." Journal of Hunan University of Technology (2015).

5. Ahearn, Mary Clare, W. Armbruster, and R. Young. "Big Data's Potential to Improve Food Supply Chain Environmental Sustainability and Food Safety." International Food \& Agribusiness Management Review (2016).

6. Zage, David, K. Glass, and R. Colbaugh. "Improving supply chain security using big data." IEEE International Conference on Intelligence and Security Informatics IEEE, (2013):254-259.

7. Zhao, S., and X. Yang. "Food safety risk assessment in whole food supply chain based on catastrophe model." Advance Journal of Food Science \& Technology 5.12(2013):1557-1560.

8. Wang, Jing, and H. Yue. "Food safety pre-warning system based on data mining for a sustainable food supply chain." Food Control 73(2017):223-229.

9. Leblanc, Denyse I., et al. "A national produce supply chain database for food safety risk analysis." Journal of Food Engineering 147(2015):24-38. 